\title{
A SIMPLE, UNIFYING MODEL OF THE EXTREME ULTRAVIOLET SPECTRA OF DWARF NOVAE IN OUTBURST
}

\author{
Christopher W. Mauche ${ }^{1}$
}

RESUMEN

Se describe brevemente un morlelo simple de la capa limítrofe y del viento del disco de acreción de novas enanas on explosión aplicándolo a los espectros dr WZ Sge y SS Cyg en explosión obtenidos con la Rejilla de Transmisión de Baja Energía de Chandra.

\section{ABSTRACT}

A simple model of the boundary layer and accretion disk wind of dwarf novae in outburst is briefly described and applied to the Chandra Low Energy Transmission Grating spectra of WZ Sge and SS Cyg in outburst.

Key Words: BINARIES: CLOSE - NOVAE, CATACLYSMIC VARIABLES - STARS: INDIVIDUAL (SS CYG, WZ SGE) — STARS: WINDS, OUTFLOWS - ULTRAVIOLET: STARS

\section{INTRODUCTION}

According to simple theory, the boundary layer between the accretion disk and the surface of the white dwarf is the dominant source of high energy rudiation in nommagnetic cataclysmic variables. When the mass-accretion rate is high, as in novalike variables and dwarf novae in outburst, the boundary layer is optically thick and its temperature is of order the blackbody temperature $T_{\mathrm{bb}}=$ $\left(G M M_{\mathrm{wd}} M / 8 \pi \sigma f R_{\mathrm{wcl}}^{3}\right)^{1 / 4} \sim 100 \mathrm{kK}$. Such a compact source of (xtrence ultriviolet (EUV; $\lambda \sim 100 \AA$ ) flux is not inconsistent. with low inclination systems like SS ( $y g\left(i \sim 40^{\prime \prime}\right)$ and VW Hyi $\left(i \sim 60^{\circ}\right)$, but the lack of EUV relipses in high inclination systems like UX UMa ( $i \approx 71^{\circ}$; Wood, Naylor. \& Marsh 1995; Pratt et al. 2004) and OY ('ir $\left(i \approx 83^{\circ}\right.$; Naylor et al. 1988; Pratt et al. 1999; Mauche \& Raymond 2000) requires an additional extended sonre of EUV flux. A plausible explanation for the origin of this extended EUV flux is proposed by Mauche \& Raymond (2000), who modeled the Ertrome liltrainolet Explorer (EUIE) spectrum of OY ('ar in superoutburst with a simple model, whercin EUV radiation from the boundary layer is scattered into the line of sight by the system's accretion disk wind. Characterizing the boundary layer spectrum as an absorbed blackbody and the wind as an expanding spherical shell, good fits were obtained with an assumed wind mass-loss rate $\dot{M}_{\mathrm{w}} \approx 1 \times 10^{-10} \mathrm{M} \ldots \mathrm{vr}^{\cdots 1}$ and a boundary laver fractional emitting area $f \approx 0.17 \cdot 1$ with a boundary layer temperature $T_{\mathrm{b} 1} \approx 95-127 \mathrm{kK}$. an absorbing column density $N_{\mathrm{H}} \approx 1.6-2.5 \times 10^{19} \mathrm{c}^{\mathrm{cm}^{-2}}$. and a luminosity $L_{\mathrm{bl}}=4 \pi f R_{\mathrm{wd}}^{2} \sigma T_{\mathrm{bl}}^{4} \approx 1 \cdot 10 \times 10^{34} \mathrm{erg} \mathrm{s}^{-1}$.

\footnotetext{
${ }^{1}$ Lawrence Livermore National Laboratory, IS
}

\section{LETG SPECTRA OF WZ SGE AND SS CYG}

Given the success of this simple model to explain the EUVE spectrum of OY Car, we used it to model the Chandra Low Energy Transmission Grating (LETG) spectra of WZ Sge and SS Cyg. The WZ Sge spectrum was obtained on 2001 July 27 during the early decline of a superoutburst that began four days earlier (Kuulkers et al. 2002), while the SS Cyg spectrum was obtained on 2001 January 16 during the plateau phase of a normal (asymmetric) wide outburst that began fours days earlier. The resulting background-subtracted, fluxed spectra are shown by the black histograms in Figure 1. The LETG spectrum of WZ Sge, which is very similar to the EUVE spectrum of OY Car, contains numerous broad emission lines, the strongest of which are asymmetric, with flux missing from the blue sides of the lines. A similar asymmetry was seen in the lines in the EUVE spectrum of OY Car, but it is much more apparent in this higher resolution (FWHM $=0.05 \AA$ for the Chandra LETG spectrometer compared to $\mathrm{FWHM}=0.5 \AA$ for the EUVE SW spectrometer) and higher signal-to-noise ratio LETG spectrum of $\mathrm{WZ}$ Sge. That these are $\mathrm{P}$ Cygni-type profiles is demonstrated clearly by the similarity of the O VI $2 s-n p(n=3.4 .5)$ lines in the EUV to the O VI $2 s-$ $2 p$ line in the FUV (Long et al. 2003). In contrast to the EUV spectra of OY Car and WZ Sge, the EUV spectrum of SS Cyg consists of a broad $(\lambda \approx 40$ $130 \hat{A}$ ) continuum, which we argue below is cut by a multitude of broad, blueshifted absorption lines.

Details of the analysis and interpretation of these spectra will be presented elsewhere (Wheatley \& Mauche 2004: Mauche 2004), but we report here the 


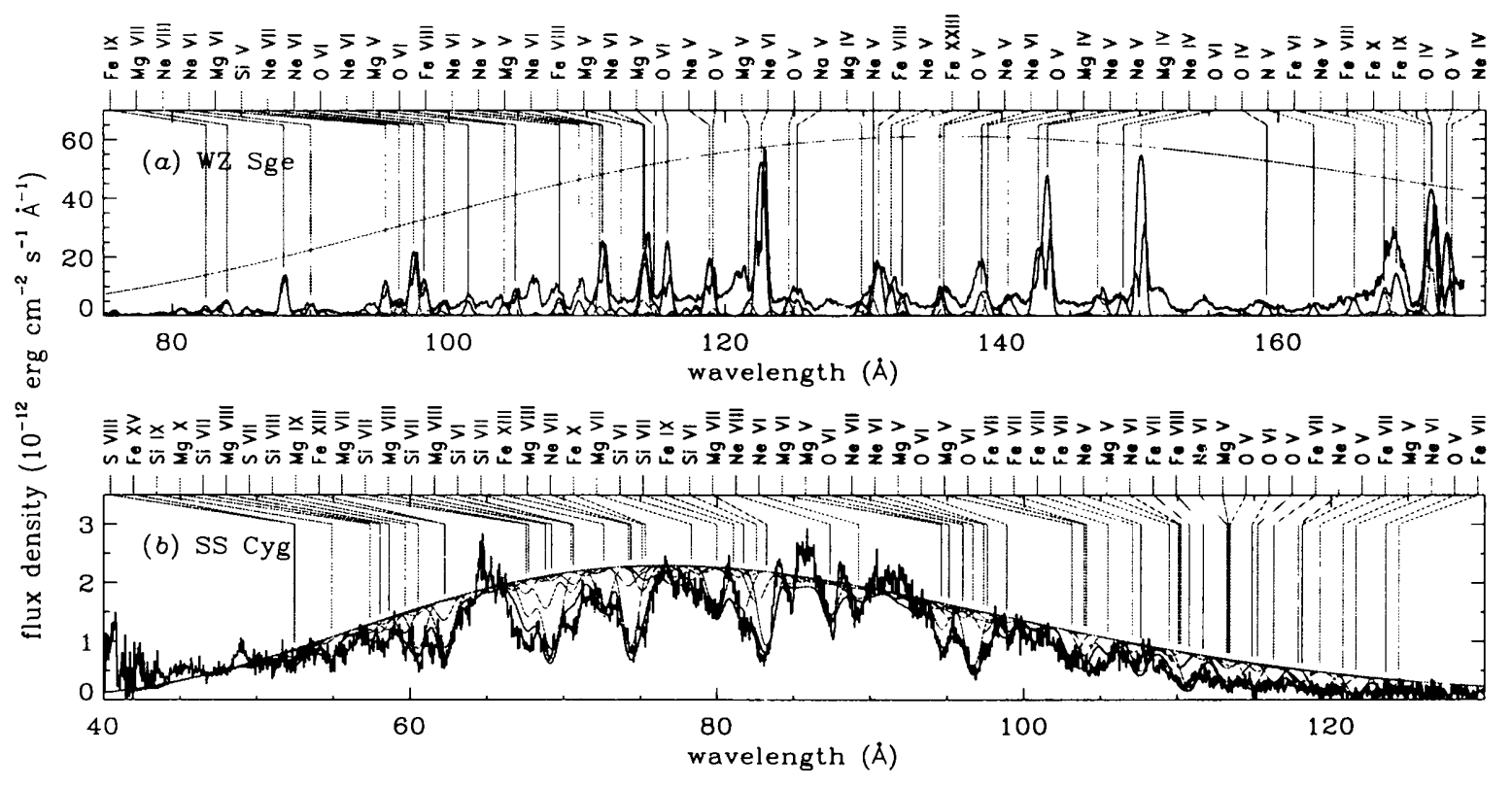

Fig. 1. Chandra LETG spectra of (a) WZ Sge in superoutburst and (b) SS Cyg in outburst (black histograms), the absorbed blackbody continua (smooth gray curves), the individual ion spectra (gray curves), and the net model spectrum (thick gray curves). The strongest lines in the models are labeled.

reasonable success we have had applying the simple scattering model developed for OY Car to WZ Sge and SS Cyg. For WZ Sge $\left(i \approx 75^{\circ}\right)$, like OY Car, we assume that the boundary layer is hidden from direct view by the accretion disk, but that its flux is scattered by the wind into the line of sight; for SS Cyg $\left(i \sim 40^{\circ}\right)$, we assume that the boundary layer is observed directly, but that its flux is scattered by the wind out of the line of sight. As shown by the gray curves in Figure 1, we obtain reasonable fits to the WZ Sge spectrum assuming a source distance $d=43 \mathrm{pc}$ and a white dwarf mass $M_{\mathrm{wd}}=1 \mathrm{M}_{\odot}$ (hence $R_{\mathrm{wd}}=5.5 \times 10^{8} \mathrm{~cm}$ ) with $T_{\mathrm{bl}}=150 \mathrm{kK}$, $f=0.13$ (hence $L_{\mathrm{bl}}=1.4 \times 10^{34} \mathrm{erg} \mathrm{s}^{-1}$ ), $N_{\mathrm{H}}=$ $1.2 \times 10^{19} \mathrm{~cm}^{-2}$, and $\dot{M}_{\mathrm{w}}=1 \times 10^{-10} \mathrm{M}_{\odot} \mathrm{yr}^{-1} ;$ and to the SS Cyg spectrum assuming a source distance $d=160 \mathrm{pc}$ and a white dwarf mass $M_{\mathrm{wd}}=1.2 \mathrm{M}_{\odot}$ (hence $R_{\mathrm{wd}}=3.9 \times 10^{8} \mathrm{~cm}$ ) with $T_{\mathrm{bl}}=250 \mathrm{kK}$, $f=0.012$ (hence $L_{\mathrm{bl}}=5.0 \times 10^{33} \mathrm{erg} \mathrm{s}^{-1}$ ), $N_{\mathrm{H}}=$ $5.0 \times 10^{19} \mathrm{~cm}^{-2}$, and $\dot{M}_{\mathrm{w}}=3 \times 10^{-11} \mathrm{M}_{\odot} \mathrm{yr}^{-1}$, with a $2500 \mathrm{~km} \mathrm{~s}^{-1}$ blueshift of the lines. Based on these results, we conclude that the boundary layer of SS Cyg in outburst is hotter, but smaller and less luminous than that of WZ Sge and OY Car in superoutburst. The worst deficiencies of the model are its inability to reproduce the blue asymmetry of the emission lines in WZ Sge, and the apparent underestimate of the level of the continuum in SS Cyg. We are developing a more sophisticated Monte Carlo model of the radiation transfer in an accretion disk wind that will hopefully remedy these problems.

Support for this work was provided in part by NASA through Chandra Award Number GO12023A issued by the Chandra X-ray Observatory Center, which is operated by SAO for and on behalf of NASA under contract NAS8-39073. This work was performed under the auspices of the U.S. Department of Energy by University of California Lawrence Livermore National Laboratory under contract No. W-7405-Eng-48.

\section{REFERENCES}

Kuulkers, E., Knigge, C., Steeghs, D., Wheatley, P. J., \& Long, K. S. 2002, in The Physics of Cataclysmic Variables \& Related Objects, ed. B. T. Gänsicke, K. Beuermann, \& K. Reinsch (San Francisco: ASP), 443 Long, K. S., Froning, C. S., Gänsicke, B., Knigge, C., Sion, E. M., \& Szkody, P. 2003, ApJ, 591, 1172

Mauche, C. W. 2004, in preparation

Mauche, C. W., \& Raymond, J. C. 2000, ApJ, 541, 924

Naylor, T., et al. 1988, MNRAS, 231, 237

Pratt, G. W., Hassall, B. J. M., Naylor, T., Wood, J. H., \& Patterson, J. 1999, MNRAS, 309, 847

Pratt, G. W., Mukai, K., Hassall, B. J. M., Naylor, T., \& Wood, J. H. 2004, MNRAS, in press

Wheatley, P. J., \& Mauche, C. W. 2004, in preparation

Wood, J. H., Naylor, T., \& Marsh, T. R. 1995, MNRAS, 274,31

Christopher W. Mauche: Lawrence Livermore National Laboratory, L-473, 7000 East Avenue, Livermore, CA 94550, US (mauche@cygnus.llnl.gov). 